\section{Rock Fracture via Strain-Softening FinITE ELEMENTS}

By Zdeněk P. Bažant, ${ }^{1}$ F. ASCE and Byung H. Oh, ${ }^{2}$ A. M. ASCE

ABstaAcr: The fracture of rock is assumed to arise from propagation of a blunt crack band with continuously distributed (smeared) microcracks or continuous cracks. This approach, justified by material heterogeneity, is convenient for finite element analysis, and allows analyzing fracture on the basis of triaxial stressstrain relations which cover the strain-softening behavior. A simple compliance formulation is derived for this purpose. The practical form of the theory involves two independent material parameters, the fracture energy and the tensile strength. The width of the crack band front is considered as a fixed material property and can be taken as roughly five-times the grain size of rock. The theory is shown to be capable of satisfactorily representing the test data available in the literature. In particular, good fits are demonstrated for the measured maximum loads, as well as for the measured resistance curves (R-curves). Statistical analysis of the deviations from the test data is also presented.

\section{INTRODUCTION}

The test results on fracture of rock, on a scale that is not very large compared to the size of inhomogeneities, show significant deviation from linear fracture mechanics $(16,17,23,39-41)$. Various methods to describe this phenomenon, consisting chiefly in adaptations of ductile fracture mechanics and the method of resistance curves (R-curves) $(1,26$ $28,35,43$ ), have been proposed. However, there seems to exist no model which could represent the gradual strain-softening caused by disperse microcracking of rock, and which would be particularly designed for use in finite element codes. The development of such a model, paralleling a previous, similar work for concrete (12), is the principal objective of this paper. The model will be calibrated and verified by comparisons with test data from the literature and the results will be subjected to statistical analysis-an important aspect for materials of great random variability such as rock.

From the physical point of view, the fracture properties of the material will be characterized in the present model not only by the fracture energy, but also by two additional parameters - the tensile strength and a certain characteristic length. The idea of introducing such further parameters is not new and dates back to the works of Dugdale (19) and Barenblatt (1-2). It is also commonly understood that the use of material parameters of this kind implies a yielding zone or fracture process zone of a finite size. In ductile fracture of plastic metals, the yielding (nonlinear) zone is large but the fracture process zone is normally assumed

${ }^{1}$ Prof. of Civ. Engrg. and Dir., Center of Concrete and Geomaterials, Technological Inst., Northwestern Univ., Evanston, Ill. 60201.

${ }^{2}$ Visiting Scholar, Dept. of Civ. Engrg., Northwestern Univ., Evanston IIl. 60201; Visiting Research Engr., Portland Cement Assoc., Skokie, Ill. 60077; presently, Asst. Prof. of Civ. Engrg., Seoul National Univ., Seoul, Korea.

Note.-Discussion open until December 1, 1984. To extend the closing date one month, a written request must be filed with the ASCE Manager of Technical and Professional Publications. The manuscript for this paper was submitted for review and possible publication on June 6, 1983. This paper is part of the Jourmal of Engineering Mechanics, Vol. 110, No. 7, July, 1984. OASCE, ISSN 0733-9399/ 84/0007-1015/\$01.00. Paper No. 18979. negligible. If the latter zone is finite, one needs to model strain-softening. This was originally done by postulating for the crack extension line a stress-displacement relation with a gradual decline of stress to zero instead of a plateau followed by a sudden stress drop. Such an approach was introduced by Knauss (28), Wnuk (43), and Kfouri, et al. (26-27). For concrete, a line crack model of this type was introduced in finite element analysis by Hillerborg and coworkers $(22,36)$. As far as the overall response is concerned, the present model is approximately equivalent to Hillerborg's model, if the transverse displacement accumulated from transverse normal strains across the crack band representing the fracture process zone is made equal to the displacement used in the stress-displacement relation for the crack line. Nevertheless, the present approach, based on a strain-softening constitutive relation, is advantageous for numerical implementation in finite element codes. Moreover, this approach makes it possible to translate into fracture modeling certain effects known from stress-strain relations.

\section{Hypothesis of Blunt Crack Band}

Following an approach initiated in 1979 for concrete $(7-9,12)$, we propose to model the front of a fracture in rock as a band of parallel cracks (or microcracks) that are continuously distributed (smeared) over a certain characteristic width, $w_{c}$. The salient feature of this approach is that $w_{c}$ is regarded as a material property. This is necessary for the smeared cracking: firstly because otherwise the strain-softening concept would not lead to results that are objective in the sense of being independent from the choice of element size (7) (except for a negligible numerical error that converges to zero); and, secondly, because analysis of strainlocalization instability $(4,6)$ shows that, in a continuum, the width of the crack band front always localizes into the smallest width permitted by the continuum smearing of a heterogeneous microstructure. The computationally convenient hypothesis of blunt crack band rests on the following two physical justifications.

Justification 1.-The stresses and strains with which we deal in any practical problem are not the actual stresses and strains at a point of the material but the stresses and strains in an equivalent homogeneous continuum which smears the inhomogeneous microstructure and averages the properties of the actual material over a certain characteristic volume (which is called the representative volume in the language of the statistical theory of randomly heterogeneous media). The representative volume must be sufficiently large compared to the maximum size of the inhomogeneities, i.e., the maximum grain size, in case of intact rock. For acceptable representation, the representative volume must be taken at least several times the grain size. Within shorter distances, the detailed distribution of stresses and strains would require statistical treatment and is not of great interest. Thus, if the width of the crack band, $w_{c}$, is not more than several times the grain size, there could be no significant differences from the line crack model in the representation of the actual behavior of rock [see Fig. 1(a,b)].

Another consequence of the material heterogeneity is that the crack path is not a straight line or a smooth curve but a highly tortuous line 


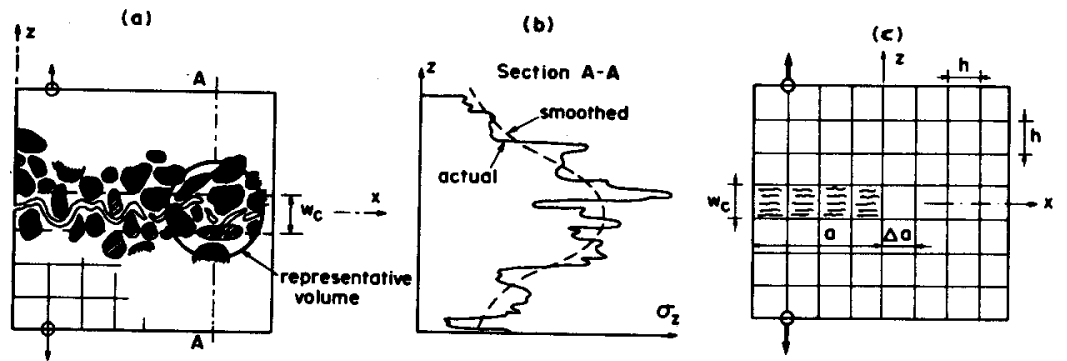

FIG. 1.-(a) Fracture in Heterogeneous Brittle Materlals, (b) Random Scatter of Actual Stresses in Microstructure and Their Continuum Smoothing, (c) Crack Band in Finite Element Mesh

swaying to each side of a straight or smoothly curved ideal crack path by distances equal to about the size of the grain. At the front, fracture is preceded by a formation of dispersed microcracks which gradually grow and join into a single major crack. The microcracks ahead of the fracture front do not lie on one straight line but deviate from such a line significantly. They seem to be concentrated along a microscopically highly tortuous line which eventually becomes the continuous fracture. It is thus geometrically obvious that a crack band of several grain sizes in width can represent the tortuous crack path and the scattered microcracks at the fracture front at least as well as a straight line [Fig. 1(a)].

Justification 2. - If the energy release rate caused by unit fracture extension is calculated from the overall energy changes in the entire structure, then the line crack model and the recently proposed crack band model give essentially the same results, provided the mesh is not too crude (i.e., at least 10 elements are used over the cross section of the domain to be solved). This fact has been numerically demonstrated for the case when the stress in the element at the fracture front drops suddenly to zero [provided the energy criterion is used (7-8)]. A demonstration has also been given for the case when the stress declines to zero gradually, over a certain distance ahead of the fracture front (see Fig. 2, Part II of Ref. 12).

Consequently, the argument whether the fracture is better represented by a line model or a crack band model is moot. The relevant distinction is that of convenience in modeling. Although Ingraffea's work (25) showed that the line crack model can be used effectively once a sophisticated finite element code has been developed, the blunt crack band model generally seems preferable for finite element modeling. In the line crack model, either a node must be split into two nodes as the fracture passes through it, or two coinciding nodes must be introduced at the outset for each node where cracking is a possibility. This increases the number of unknowns during the process of analysis, and in the former case the nodes must be renumbered if the band structure of the structural stiffness matrix should be preserved. When the direction of propagation of the fracture is not known at a certain stage of computation, the nodal coordinates must be redefined during computation and calculations must be run for various conceivable locations of the node into which the fracture front extends, so as to be able to select among these locations the correct one.

By contrast, neither doubling of the nodes nor their renumbering is needed in the blunt crack model. Satisfactory results for failure loads can be obtained by using a uniform square mesh, without any mesh refinement near the fracture front. So, the fracture front location need not be known in advance $(7-9,12)$. Furthermore, propagation of fracture in an arbitrary direction can be represented using the same fixed mesh, without redefining the coordinates of the nodes. A fracture propagating in a skew direction with regard to the mesh lines is here represented as a zigzag crack band whose overall direction characterizes the direction of the actual fracture. The direction of cracks within the band can be inclined with regard to the mesh lines, which is represented in a finite element code by using inclined directions of orthotropy for the material stiffness matrix of the cracked material.

The fact that, in the crack band model, the fracture formation is represented by means of changing the stiffness (or compliance) matrix of the material is quite convenient for computer programming. This fact also provides additional possibilities in introducing various influencing factors by means of the stress-strain relations for the crack band. It has been shown for concrete, for example (12), that the effect of the compressive normal stress in the direction parallel to the fracture can be introduced into the stress-strain relation for the crack band utilizing previously established biaxial failure envelopes for concrete. A similar approach may be possible for rock. Another possibility in the crack band model is to utilize for the time-rate effect in fracture what is known about viscoelastic stress-strain relations.

The idea of a continuous (smeared) representation of cracking in finite elements, introduced by Rashid (37), and Ngo and Scordelis (34), has been widely used since the 1960 s in large computer programs because of its simplicity. In these programs however the fracture extension has been decided on the basis of the strength criterion. It has been shown (7-9) that the use of this criterion is not objective in that the results of calculation may often depend very much on the analyst's choice of the size of the finite element, and may converge to an incorrect solution for which cracking consumes zero work and crack propagation occurs for an arbitrarily small load. This lack of objectivity (or incorrect convergence) can be remedied by adopting an energy criterion for fracture.

\section{Stiffness Matrix of fully Cracked Material}

For many practical situations it is possible to assume that the directions of the principal stresses and strains coincide and do not rotate significantly during the time interval in which the fracture front passes through a given station. Under this assumption we may characterize the stress-strain relation of the material in terms of principal stresses $\sigma_{x}, \sigma_{y}$, $\sigma_{z}$ and principal strains $\epsilon_{x}, \epsilon_{y}, \epsilon_{z}$. Subscripts $x, y$, and $z$ refer to cartesian coordinates $x_{1}=x, x_{2}=y$ and $x_{3}=z$. The crack planes are assumed to be normal to the axis, $z$. Grouping the principal stresses and strains into column matrices $\sigma=\left(\sigma_{x}, \sigma_{y}, \sigma_{z}\right)^{T}, \epsilon=\left(\epsilon_{x}, \epsilon_{y}, \epsilon_{z}\right)^{T}$, in which $T$ denotes the transpose, we may write the elastic stress-strain relation as $\sigma$ 
$=\mathbf{D} \epsilon$ in which

$\mathbf{D}=\left[\begin{array}{ccc}D_{11} & D_{12} & D_{13} \\ & D_{22} & D_{23} \\ \text { sym. } & & D_{33}\end{array}\right]$

This is the stiffness matrix of the uncracked elastic material subjected to small strains. The matrix in Eq. 1 is written in the general orthotropic form since rocks are often orthotropic.

Consider now that the material is intersected by a system of continuously distributed parallel cracks normal to axis $z$. Since there is no stiffness in the direction, $z$, normal to the cracks, the third column and the third row of the stiffness matrix must be zero. The remaining $(2 \times 2)$ diagonal submatrix must then take the form of a stiffness matrix for plane stress behavior. Accordingly, the stress-strain relation of a fully cracked material takes the form $\sigma=D^{f r} \epsilon$, in which superscript " $f r$ " refers to the fractured state and

$\mathbf{D}^{f r}=\left[\begin{array}{lll}D_{11}-D_{13}^{2} D_{33}^{-1}, & D_{12}-D_{13} D_{23} D_{33}^{-1}, & 0 \\ & D_{22}-D_{23}^{2} D_{33}^{-1}, & 0 \\ \text { sym. } & & 0\end{array}\right]$

(see e.g., Ref. 42). Note that this stiffness matrix requires all cracks to be perfectly normal to axis $z$, which is not exactly true, and that it also neglects characterizing the friction properties of inclined cracks subjected to shear. Since we, however, assume the cracks to form in the direction of the maximum principal tensile stress, the latter limitation is not serious for the front of the fracture.

\section{Compliance of Progaessively Microcracking Material}

To characterize the progressive development of microcracks in the fracture process zone (i.e., in the crack band), we need to introduce a continuous transition from the secant stiffness matrix of the uncracked material (Eq. 1) to the secant stiffness matrix of the fully cracked material (Eq. 2). This task is not very simple in the stiffness matrix formulation since every element of the stiffness matrix has to be changed. It was found (12), however, that this task becomes much easier if one considers the inverse, compliance matrix $C$. In this case the stress-strain relation is $\epsilon=\mathbf{C} \boldsymbol{\sigma}$ in which

$\mathbf{C}=\mathbf{D}^{-1}=\left[\begin{array}{ccc}C_{11} & C_{12} & C_{13} \\ & C_{22} & C_{23} \\ \text { sym. } & & C_{33}\end{array}\right]$.

At this point we should note that introduction of cracks normal to axis $z$ should have no affect on the compliances in the directions parallel to cracks; it should only cause an increased compliance for the direction normal to the cracks. This compliance should become infinite when the material is fully cracked. This suggests (12) defining the secant compliance matrix for a partially cracked material in the form
$\mathrm{C}(\mu)=\left[\begin{array}{ccc}C_{11} & C_{12} & C_{13} \\ & C_{22} & C_{23} \\ \text { sym. } & & C_{33} \mu^{-1}\end{array}\right]$

Parameter $\mu$, called the cracking parameter (12), is introduced to characterize the progressive development of microcracks normal to axis $z$.

First, we must check whether the use of the secant compliance matrix in Eq. 4 is equivalent to the use of the stiffness matrix in Eq. 2 (which is now a generally accepted practice widely used in finite element programs). That this is indeed so ensues from the following statement (theorem) which can be easily proven (12):

$\mathbf{D}^{f r}=\lim _{\mu \rightarrow 0} \mathbf{C}^{-1}(\mu)$

This means that the foregoing stiffness matrix of a fully cracked material (Eq. 2) is the limit of the inverse of the compliance matrix with cracking parameter $\mu$ (Eq. 4) as this parameter tends to 0 .

Consequently, a gradual transition from a crack-free state to a fully cracked state may be simply obtained by a continuous variation of parameter $\mu$ between the limits 1 and 0 , i.e.

uncracked: $\mu=1$; fully cracked: $\mu=0$

In practical computer programming, the fully cracked material may be conveniently characterized by setting $\mu^{-1}$ to be a very large number (e.g., $10^{30}$ ). The computer may then be left to carry out the inversion, which yields almost exactly the stiffness matrix in Eq. 2, the elements of the last row and column being of the order of $10^{-30}$ instead of zeros. This procedure saves a programmer's time.

The variation of the cracking parameter, $\mu$, can be determined either indirectly from fracture tests, or directly from the complete uniaxial tensile stress-strain diagram in which the stress is reduced to zero at increasing strain. The indirect determination of $\mu$ will be pursued here, for lack of tensile strain-softening data. Such data can be measured, as is well-known, only when a sufficiently fast servo-control is available and the testing machine is sufficiently stiff, or when the specimen is stabilized by sufficiently stiff parallel steel bars. According to their private communication in August 1983, S. P. Shah and J. Labuz of Northwestern University succeeded in directly observing strain-softening in tensile tests of granite. However, since appropriate tensile test data do not appear to exist for rock, no such results for rock seem as yet to have been published, although they have been obtained for concrete, which behaves similarly $(20-21,24,38)$ to rock. These tests clearly confirm the existence of the gradual decrease of stress at increasing strain (i.e., strainsoftening). The softening slope is normally three to four times smaller than the initial tangent modulus. Although the actual stress-strain relation appears to be smoothly curved, it is convenient to approximate it by a bilinear stress-strain diagram (Fig. 2), in which the declining (strainsoftening) segment is characterized by compliance $C_{33}^{t}$. For uniaxial tensile stress $\sigma_{z}$, we may then write

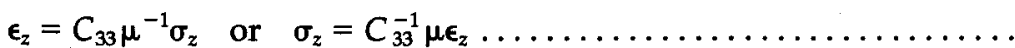




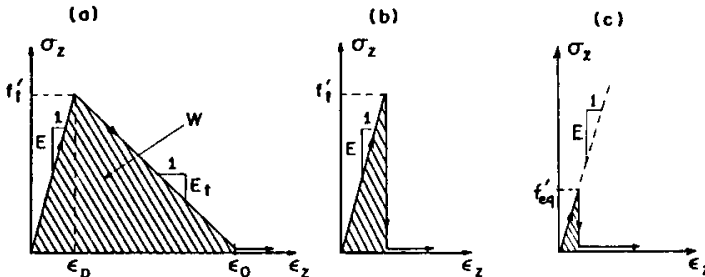

FIG. 2.-(a) Idealized Bilinear Unlaxlal Stress-Strain Relation Used to Fit Test Data (b) Stress-Strain Relation with Sudden Stress Drop (c) with a Reduced Strength Limit

This must be equivalent to the following equation for the straight line of the strain-softening branch:

$\sigma_{z}=\frac{\epsilon_{z}-\epsilon_{0}}{C_{33}^{t}}$

Here $C_{33}^{t}$ is negative and $\epsilon_{0}$ represents the terminal point of the strainsoftening branch at which the tensile stress is reduced to zero (Fig. 2). This point is related to the strain, $\epsilon_{p}$, at the peak stress point as $\epsilon_{0}=\epsilon_{p}$ $+\left(-C_{33}^{t}\right) f_{i}^{\prime}$. Comparison of Eqs. 7-8 provides

$\mu^{-1}=\frac{-C_{33}^{t}}{C_{33}} \frac{\epsilon_{z}}{\epsilon_{0}-\epsilon_{z}}$

This is the law which governs the variation of the cracking parameter, $\mu$, at increasing $\epsilon_{z}$ (loading).

For unloading and reloading, it is assumed that the stiffness and compliance matrices remain constant and the same as they were at the last maximum of $\epsilon_{z}$. If this last maximum is exceeded, then Eq. 19 for virgin loading is again followed unless $\epsilon_{z}>\epsilon_{0}$. Admittedly, this description of unloading is simplified. However, it is found that the results for maximum loads of typical fracture specimens, as well as the R-curves, depend very little on the form of the law for unloading and reloading.

The use of a straight line for the strain-softening segment avoids some more complicated questions in relating the fracture energy to the stressstrain diagram. The formation of the fracture process zone may be regarded as a strain-localization instability of a continuum. The onset of this instability depends on the tangent modulus and the unloading modulus at the current point of the stress-strain curve. If the tangent modulus varies continuously, as is the case for a smoothly curved tensile stress-strain relation, the instability that leads to fracture can initiate at various points on the strain-softening branch (for a more detailed analysis, see Eqs. 38-52 of Ref. 4). According to such an analysis, the work consumed by fracture does not necessarily correspond to the complete area under the stress-strain curve. Rather, it equals the area limited by the strain-softening branch and the unloading branch from the point on this curve at which the instability initiates, which does not have to coincide with the peak stress point. Depending on the precise point at which instability initiates, various areas corresponding to fracture en- ergy can obviously be obtained. For the bilinear stress-strain diagram considered here, however, the tangent modulus is constant along the entire strain-softening branch, and the unloading modulus decreases (which tends to stabilize the specimen as shown in Ref. 14). If the fracture should occur under such circumstances, it must occur right at the peak stress point, in which case the work consumed by fracture corresponds to the complete area under the stress-strain curve (4). Thus, the bilinear stress-strain diagram avoids ambiguity in relating the fracture energy to the stress-strain diagram, for which stability analysis would otherwise be necessary.

Many rocks, like concrete, may be treated as isotropic. The compliance and stiffness matrices for a partially cracked isotropic material then assume the following special forms:

$$
\begin{aligned}
& C(\mu)=\frac{1}{E}\left[\begin{array}{ccc}
1 & -v & -v \\
-v & 1 & -v \\
-v & -v & \mu^{-1}
\end{array}\right] \\
& \mathbf{D}^{f r}=\frac{E}{1-v^{2}}\left[\begin{array}{lll}
1 & \nu & 0 \\
\nu & 1 & 0 \\
0 & 0 & 0
\end{array}\right] \ldots
\end{aligned}
$$

in which $E=$ Young's modulus; and $\mu=$ Poisson's ratio.

The foregoing matrices are in effect secant stiffness or compliance matrices. For finite element computation, the total stress-strain relations defined by these matrices may be converted to an incremental form. This form may be obtained by differentiating the equation $\epsilon=\mathbf{C} \boldsymbol{\sigma}$, in which $\mathrm{C}$ is given by Eq. 3 or Eq. 10 . In this manner the appropriate quasi-elastic incremental stress-strain relation may be obtained.

For finite element computations, it is also necessary to enlarge the compliance or stiffness matrices to a $(6 \times 6)$ form, inserting additional three rows and columns for the shear strains and stresses. The precise values of the associated shear compliance or stiffness coefficients are not too important, since fracture is assumed to be normal to the direction of the maximum principal tensile stress, in which case the shear stress is zero. The question of shear terms, however, acquires some importance if either during the fracture process or after the completion of the fracture the subsequent loading produces relative tangential displacement across the crack band. In such a case, it is appropriate to use shear stiffness or compliance coefficients which reflect the frictional-dilatant properties of cracks $(10,15)$.

The fact that total (secant) stress-strain relations are used implies that response is path-independent. In reality, of course, all inelastic behavior is path-dependent. Nevertheless, the simplification seems to be acceptable since the principal stress directions normally do not rotate significantly during the passage of the fracture process zone through a fixed station in the material. In any case, the same assumption of a path-independent stress-strain relation in the vicinity of the fracture front is implied in the J-integral method for ductile fracture, which represents a well-substantiated and widely-used approach.

The fact that cracking is modeled solely by modifying one diagonal 
term of the compliance matrix, without any change in the off-diagonal terms, is justified by assuming all microcracks to be perfectly flat and normal to axis $z$. In reality, one must expect a certain statistical distribution of the orientations of the microcracks, the orientation normal to axis $z$ being the prevalent one. This could be modeled by adjusting the remaining two diagonal terms of the compliance matrix (Eq. 4), e.g., by dividing $C_{11}$ and $C_{22}$ in Eq. 4 by $[\alpha+(1-\alpha) \mu]$ where $\alpha$ is a coefficient for transverse damage, $0<\alpha \leq 1$. However, no test data appear to exist for determining $\alpha$. Anyhow, in simulating the fracture tests considered here, coefficient $\alpha$ would have a negligible effect because the normal stress parallel to the crack is almost zero near the crack front for all types of tests.

The use of stress-strain relations to describe the fracture process zone is advantageous in that certain effects which have already been determined in terms of the stress-strain relations may be directly introduced in fracture. Thus, determining separately the time-dependence (or ratedependence) of the stress-strain relation, it should be possible to obtain the time (or rate) effect in fracture. Also, the information known from testing the failure envelopes under combined stress states may be directly translated into fracture. For example, it is of interest to describe the effect of normal compressive stresses parallel to the cracks. It is known that such compressive stresses reduce the tensile strength in the transverse direction, and also reduce the fracture toughness in the transverse direction. The measured biaxial failure envelopes in the compressiontension range seem to consist of approximately straight lines connecting the failure points for the uniaxial tensile failure and for the uniaxial compression failure in the $\left(\sigma_{x}, \sigma_{y}\right)$ plane. Accordingly, one may suppose that the compressive stresses, $\sigma_{x}$ and $\sigma_{y}$, parallel to the cracks reduce the peak stress (Fig. 2) by the amount $\Delta f_{t}^{\prime}=k\left(\sigma_{x}+\sigma_{y}\right)$, in which $k=$ $f_{t}^{\prime} / f_{c}^{\prime}, f_{t}^{\prime}=$ the uniaxial tensile strength, and $f_{c}^{\prime}=$ the uniaxial compression strength. This then yields

for $\Delta f_{t}^{\prime} \leq 0: f_{t c}^{\prime}=f_{t}^{\prime}+\Delta f_{t}^{\prime} ;$ for $\Delta f_{t}^{\prime}>0: \quad f_{t c}^{\prime}=f_{t}^{\prime} \ldots \ldots \ldots \ldots$

in which $f_{t c}^{\prime}=$ a modified peak stress value to be substituted for $f_{t}^{\prime}$ in evaluating $\mu$ (Eq. 9).

It is also worth noting that the present treatment of progressive microcracking by reducing material stiffness with a multiplicative parameter (as in Eq. 4) bears some resemblance to the so-called continuous damage mechanics, which has recently been formulated by Loland (31), Lorrain (32), Mazars (33), and others. However, the treatment of damage is here tensorial rather than scalar. Besides, there exists a fundamental difference: The concept of damage is here considered to be inseparable from a zone of a certain characteristic width, $w_{c}$, to which we now turn attention.

\section{Fracture Parameters}

The fracture energy is the energy consumed by the formation of all cracks in the crack band per unit length of the band (and unit thickness). It may be calculated as

$G_{f}=W_{f} w_{c}$ in which $w_{c}=$ width of the crack band front (fracture process zone); and $W_{f}=$ work of the tensile stress = area under the tensile stress-strain curve (Fig. 2), i.e.

$W_{f}=\int_{0}^{\epsilon_{0}} \sigma_{z} d \epsilon_{z}$

According to the starting assumptions, $w_{c}$ should be the smallest distance on which the heterogeneous aggregate material may be treated as an equivalent homogeneous continuum. This condition however does not yield a sufficiently precise value of $w_{c}$. In theory, it should be possible to determine the crack band width by analyzing, on the basis of loading and unloading tangent moduli, the strain-localization instability that leads to fracture (similarly to Refs. 3 and 14). Such an analysis would, however, be quite complicated. Therefore, $w_{c}$ has been determined empirically by optimizing the fits of fracture test data.

For the bilinear tensile stress-strain relation (Fig. 2), we have

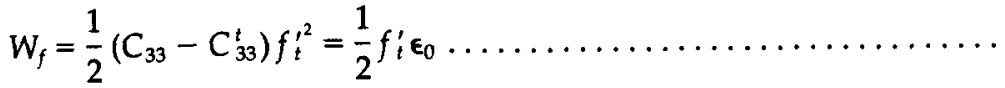

from which we obtain

$w_{c}=\frac{2 G_{f}}{f_{t}^{\prime 2}} \frac{1}{C_{33}-C_{33}^{t}}$.

in which $C_{33}^{t}$ is negative. This equation indicates that $w_{c}$ may be determined by obtaining, from the condition of optimum fit of fracture test data, the tensile strength, the fracture energy, and the softening compliance. It was this procedure which was applied to generate the data fits reported here.

From the last relation we also see that the uncertainty in $w_{c}$ is related to that in the softening compliance. If certain test data can be fitted well with different softening compliances, the value of $w_{c}$ is not too important. This has been the case for some data. However, the value of the softening compliance, $C_{33}^{t}$, affects quite strongly the length of the fracture process zone ahead of the fracture front, and if this length follows from the available data unambiguously, so does $w_{c}$. The length of the fracture process zone becomes unimportant for sufficiently large structures for which it is small compared to the dimension of the cross section. In such a case, the value of $w_{c}$ can be quite arbitrarily chosen, preserving, of course, the correct value of the fracture energy.

To assure that $C_{33}^{t}$ be negative, Eq. 16 with $C_{33}=1 / E$ indicates that the following condition must be met

$w_{\mathfrak{c}}<w_{0}, \quad w_{0}=\frac{2 G_{f} E}{f_{t}^{\prime 2}}$

When $w_{c}=w_{0}$, the stress-strain diagram has a vertical straight drop of stress to zero [Fig. 2(b)].

If the use of finite elements of the size $w_{c}$ would lead to too many elements, as in the case of a relatively large rock mass, then the finite element size may be increased, along with $w_{c}$, if a larger $\left|C_{33}^{t}\right|$ (steeper 


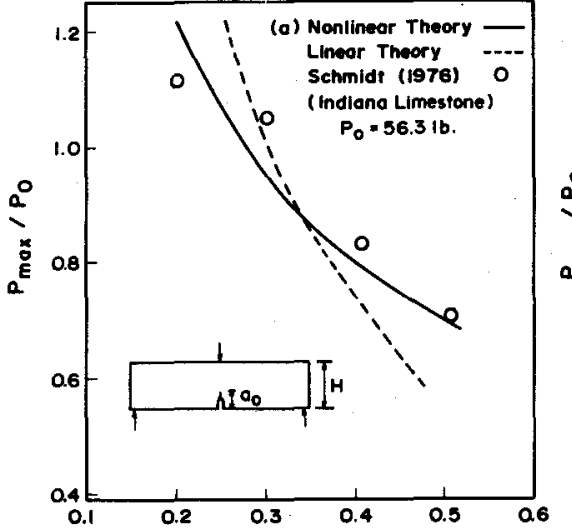

Rel. Initial Crock Length $\left(\mathrm{O}_{0} / \mathrm{H}\right)$

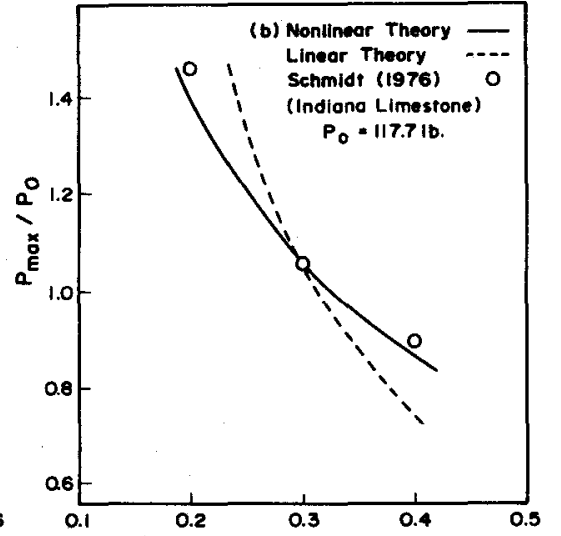

Rel. Initial Crack Length $\left(a_{0} / H\right)$

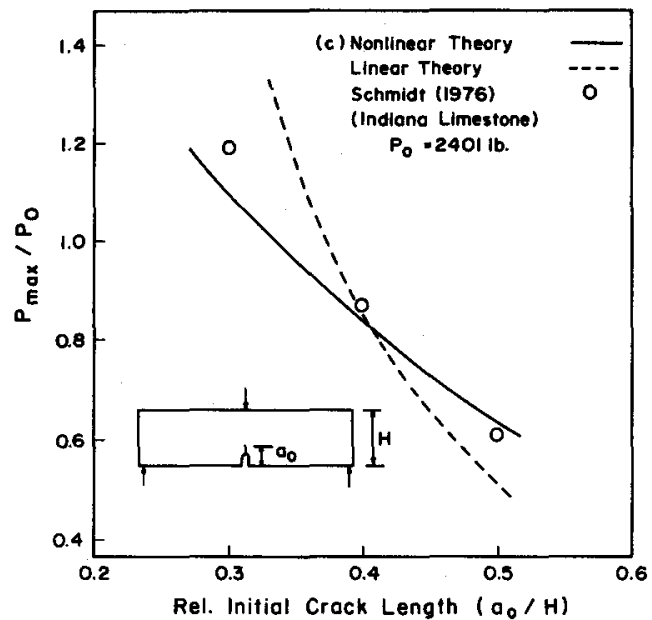

FIG. 3.-Fits of the Maximum Load Data of Schmidt (1976) for Indiana Limestone

strain-softening) is considered as required by Eq. 16 (Fig. 2). This is, however, limited by Eq. 17, and a further increase in the finite element size (i.e., in $w_{c}$ ) cannot be obtained.

If the rock mass is so large that still larger finite elements are needed, then it is possible to modify not only the declining slope to a vertical one, but also to reduce the strength limit, $f_{t}^{\prime}$, provided that the correct value of the fracture energy $G_{f}$ is preserved. That is, we must preserve the relation $G_{f}=h f_{t}^{\prime 2} / 2 E$, in which $h$ is the size of square finite elements in a regular square mesh. According to this relation, we must then use the strength limit

$f_{e q}^{\prime}=\sqrt{\frac{2 G_{f} E}{h}}$

which represents what has previously been introduced as the equivalent tensile strength (7-9). It has been verified that for large structures in which many finite elements (approximately, over 12) are laid across the cross section, the fracture analysis with the equivalent strength according to Eq. 18 gives essentially the same results as the fracture analysis based directly on fracture energy. The results are then also approximately the same as those obtained from linearly elastic fracture mechanics. In this case, the tensile strength value and the width of the fracture front become irrelevant. Thus, the theory presented here has the purpose of extending fracture analysis to regions of smaller size as compared to inhomogeneities of the material.

It has been verified numerically (12) that the width of the elementwide crack band for finer mesh subdivisions and larger structures (compared to aggregate size) can be chosen rather arbitrarily if the strainsoftening slope is properly adjusted.

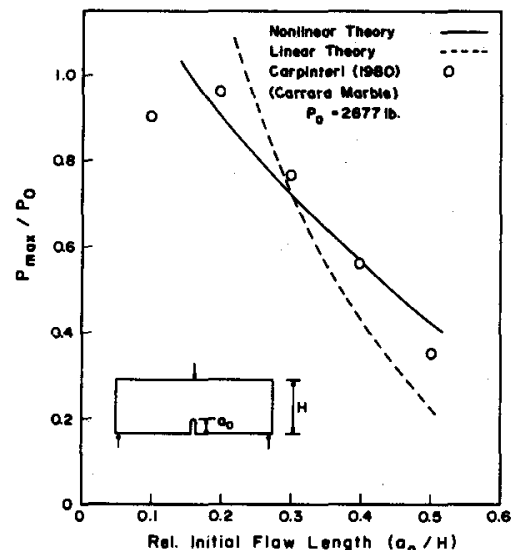

FIG. 4.-Fits of the Maximum Load Data of Carpinterl (1980) for Carrara Marble

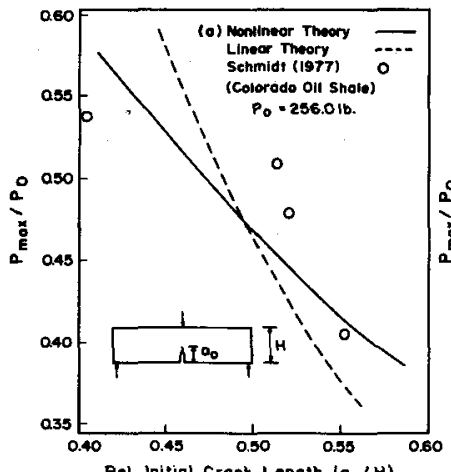

(a)

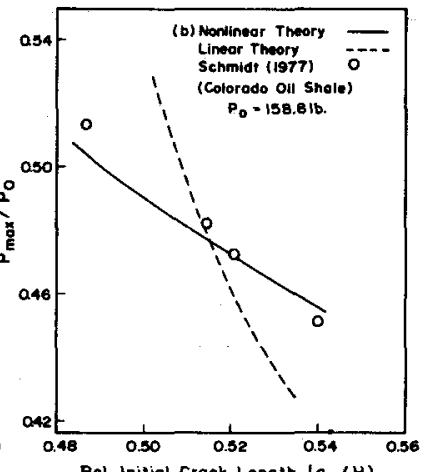

Rel Initiol crack Length $(0,1)$
FIG. 5.-Fits of the Maximum Load Data of Schmidt (1977) for Colorado Oil Shale 


\section{Fitting of Test Data}

The mathematical model just described, which was originally developed and successfully applied for concrete (12), has been fitted to various test data on rock fracture available in the literature. The optimum fits attained are plotted as the solid lines in Fig. 3-7. For comparison, the linear elastic fracture mechanics fits that are optimum in the least-square sense are also shown in these figures (the dashed curves). The material parameters corresponding to all the fits are summarized in Table 1. The

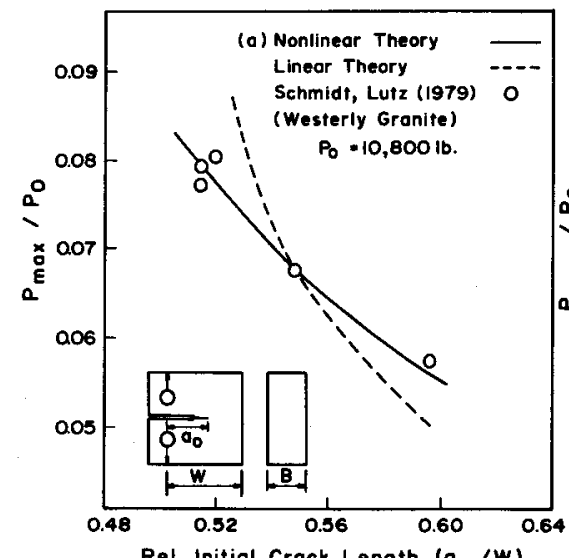

Rel. Initiol Crack Length $\left(a_{0} / w\right)$

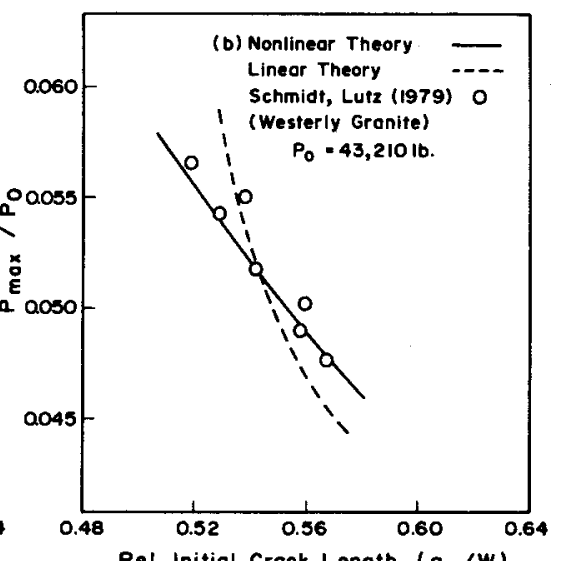

Rel. Initiol Crock Length $\left(a_{0} / w\right)$

\section{FIG. 6.-Fits of the Maximum Load Data of Schmidt and Lutz (1979) for Westerly} Granlte
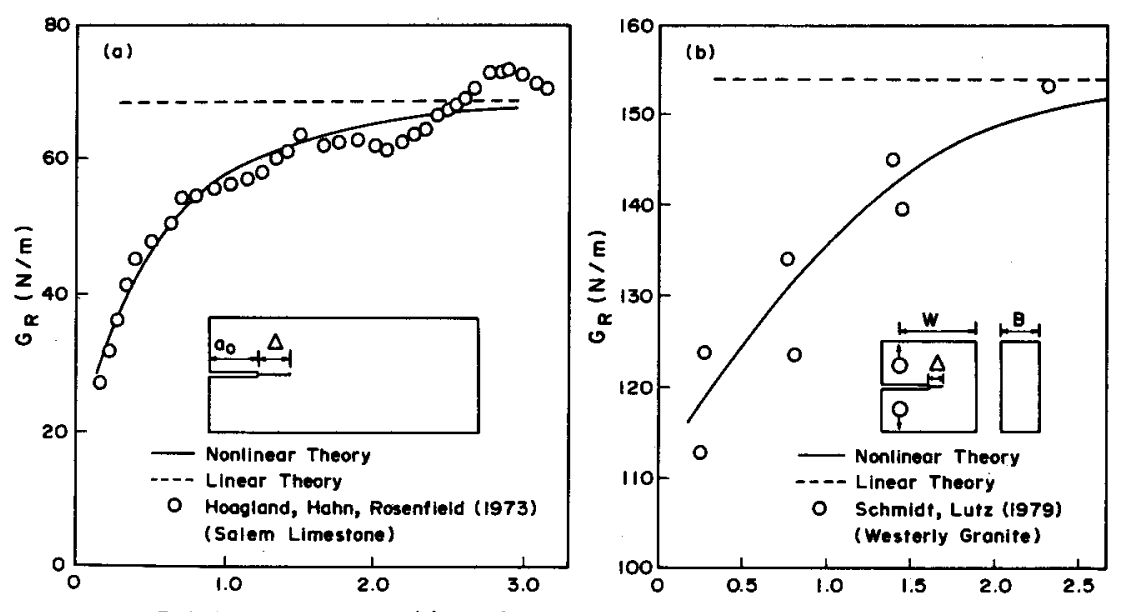

Rel. Crack Extension $\left(\Delta / w_{c}\right)$

FIG. 7.-Apparent Fracture Energy versus Crack Extension According to Hoagland et al., (1973) for Salem Limestone, and Schmidt and Lutz (1979) for Westerly Granite

TABLE 1.-Parameters for Test Data Fits

\begin{tabular}{|c|c|c|c|c|c|c|c|}
\hline \multicolumn{2}{|l|}{$\begin{array}{l}\text { Test series } \\
\text { (1) }\end{array}$} & $\begin{array}{c}f_{i}^{\prime}, \text { in } \\
\text { pounds } \\
\text { per square } \\
\text { inch } \\
\text { (2) }\end{array}$ & $\begin{array}{l}E \text {, in } \\
\text { kips per } \\
\text { square } \\
\text { inch } \\
\text { (3) }\end{array}$ & $\begin{array}{l}G_{f}, \text { in } \\
\text { pounds } \\
\text { per } \\
\text { inch } \\
(4)\end{array}$ & $\begin{array}{c}d_{g}, \text { in } \\
\text { inches } \\
(5)\end{array}$ & $\begin{array}{c}w_{c} \text {, in } \\
\text { inches } \\
(6)\end{array}$ & $\begin{array}{c}G_{f}^{\text {lin }}, \text { in } \\
\text { pounds } \\
\text { per } \\
\text { inch } \\
(7)\end{array}$ \\
\hline 1. Schmidt (1976) & No. 1 & $356^{*}$ & $3,130^{*}$ & $0.068^{*}$ & 0.0787 & $0.3935^{*}$ & $0.027 *$ \\
\hline 2. & 2 & $356 *$ & $3,130^{*}$ & $0.068^{*}$ & 0.0787 & $0.3935 *$ & $0.186^{*}$ \\
\hline 3. & 3 & $356^{*}$ & $3,130^{*}$ & $0.068 *$ & 0.0787 & $0.3935 *$ & $1.751^{*}$ \\
\hline 4. Schmidt (1977) & No. 1 & $768^{*}$ & $3,600^{*}$ & $0.121 *$ & 0.0197 & $0.0985^{*}$ & $0.162 *$ \\
\hline 5. & & $477 *$ & $2,232 *$ & $0.076^{*}$ & 0.0197 & $0.0985 *$ & $0.168^{*}$ \\
\hline 6. Carpinteri & & 725* & $2,200^{*}$ & $0.392 *$ & 0.0079 & $0.0395 *$ & $0.530^{*}$ \\
\hline 7. Schmidt, Lutz & No. 1 & $1,394^{*}$ & $3,300^{*}$ & $0.874^{*}$ & 0.0296 & $0.1480 *$ & $1.697^{*}$ \\
\hline 8. & & $1,394 *$ & $3,300^{*}$ & $0.874 *$ & 0.0296 & $0.1480 *$ & $0.806^{*}$ \\
\hline 9. & 3 & $1,394^{*}$ & $3,300^{*}$ & $0.874 *$ & 0.0296 & $0.1480 *$ & $0.879 *$ \\
\hline $\begin{array}{l}\text { 10. Hoagland, Hah } \\
\text { Rosenfield }\end{array}$ & & $427^{*}$ & $2,000^{*}$ & $0.374^{*}$ & 0.0787 & $0.3940 *$ & $0.390^{*}$ \\
\hline
\end{tabular}

*Asterisk indicates numbers estimated by calculations; without asterisk-as reported; and $G_{f}^{\text {lin }}=G_{f}$ values for the linear theory fits.

Note: $\mathrm{psi}=6,895 \mathrm{~N} / \mathrm{m}^{2}, \mathrm{lb} / \mathrm{in} .=175.1 \mathrm{~N} / \mathrm{m}$, in. $=25.4 \mathrm{~mm}, \mathrm{ksi}=1,000 \mathrm{psi}$.

test specimens were assumed in all cases to be in a plane stress state, $\sigma_{y}=0$. All fits were calculated by the finite element method, using a regular square mesh of identical four-node finite elements each of which consists of four constant-strain triangles. The stress-strain relation just developed has been assumed to apply for all the finite elements but using small enough loading steps the strain softening regime was reached only within one row of finite elements. The tangent stiffness coefficients were assumed to be the same for all the four triangles composing a square element and were determined from the average of the strains in these four triangles.

Based on our initial arguments relative to homogeneous continuum smoothing of a randomly heterogeneous material, the width of the element-wide blunt crack band front should not be considered less than about $w_{c}=3 d_{a}$ in which $d_{a}$ is the maximum size of the inhomogeneities (the grain size in rock, or the aggregate size in concrete). On the other hand, with regard to the analysis of strain-localization instability in a tensile test specimen $(3,4,14)$, $w_{c}$ should not be much larger than $3 d_{a}$. In a preceding analysis (12) of 22 series of concrete fracture tests reported in the literature - quite a large statistical sample-it was found that for all normal concretes the optimum width of the crack band front (fracture process zone) which gives the best fits of the test data is roughly $w_{c}=$ $3 d_{a}$ (this was concluded by comparing the fits for $w_{c}=2 d_{a}, 3 d_{a}, 4 d_{a}, 6 d_{a}$, etc.).

With this result in mind, the fits of all rock fracture data analyzed here have also been sought under the restriction that the ratio $w_{c} / d_{g}$ (where $d_{g}=$ grain size) be the same for all test data for the various rocks considered. Thus, the fitting of the test data was carried out under the condition that only the values of $G_{f}$ and $f_{t}^{\prime}$ (and of the elastic constants) may vary from rock to rock (Table 1 ). Various values of the ratio $w_{c} / d_{g}$ were tried, and optimum fits were obtained for 
$w_{c}=5 d$

This value applies to very different types of rock (Figs. 3-7).

This result, however, should be considered as tentative and the possibility that still other rocks might require for best fits other ratios $w_{c} / d_{g}$ must not be ruled out. At present, much fewer test data exist in the literature for nonlinear fracture of rock than of concrete.

Due to the last relation, as well as the energy relation in Eq. 15, the present nonlinear fracture theory is a two-parameter theory. The two material parameters to be determined by fitting test data are $G_{f}$ and $f_{t}^{\prime}$.

From microscopic observations of the microcrack band ahead of the crack tip, it is known that the width of this band increases as the crack extends and the density of microcracks varies across the width of the band. This is not reflected in Eq. 19. However, $w_{c}$ should not be regarded as the actual width of the microcracking zone but as some effective or equivalent width needed to obtain the correct energy consumption.

The test data in Figs. 3-6, obtained by Schmidt, Lutz, and Carpinteri for Indiana limestone, Carrara marble, Colorado oil shale, and Westerly granite, are presented as plots of the maximum load, $P_{\max }$, measured in the fracture test, versus the length of notch $a_{0}$. The $P_{\max }$ values were non-dimensionalized as the ratios $P_{\max } / P_{0}$ in which $P_{0}=$ the maximum load that is obtained from the bending theory (i.e., for a linear stress distribution throughout the ligament). In Fig. 3, $P_{0}=2 D H^{2} f_{t}^{\prime} / 3 L=\max$ imum load based on a bending theory calculation for an uncracked beam, where $H=$ beam depth, $D=$ beam thickness, $L=$ beam span, and $f$ $=$ the direct tensile strength. The same definition of $P_{0}$ was used in Figs. 4-5. For the data in Fig. 6, $P_{0}=W D f_{t}^{\prime}$ in which $W=$ specimen width, and $D$ its thickness.

The finite element calculation was carried out in small loading steps, controlling the displacement increments at the loading points, calculating at each load increment the reaction at the loading point, and then identifying the maximum value among these reactions. The dashed curves pertaining to linear fracture mechanics were actually calculated also by the same finite element code using the previously published blunt crack band approach $(7,8)$, in which a sudden stress drop and a finite element version of the energy release criterion is considered. With a sufficiently fine mesh, this analysis yields results which do not differ from the exact linear fracture mechanics solutions by more than $1 \%-2 \%$.

In judging the fits achieved with the present theory (solid lines in Figs. 3-6), comparison should be made with the dashed lines representing the best possible fits by linear elastic fracture mechanics. It is clear that the improvement is significant.

The data by Hoagland, et al. (23), and by Schmidt and Lutz (4) (which are shown in Fig. 8), represent the R-curves (resistance curves) in which the apparent fracture energy determined in the test is plotted as a function of the crack extension from a notch in slow, stable crack growth. The present theory is also capable of predicting the R-curves, and the fits obtained with the present theory are shown in Fig. 7 as the solid lines. The reason is that at the beginning of the crack extension, the zone that undergoes strain-softening is small, and therefore little energy is being consumed. The full value of energy consumption, which corresponds to the final asymptotic value of fracture energy, $G_{f}$, is obtained only when the fracture process zone length develops fully, in which case the stress at the tip of the notch drops to zero (for further explanation see Ref. 12). The classical linear elastic fracture mechanics predicts no R-curve, since the fracture energy is considered as a constant. Thus, the dashed lines are plotted in Fig. 7 as horizontal straight lines, made to pass through the terminal measured value at which the apparent fracture energy value stabilizes.

The capability of the present theory to predict the R-curve on the basis of only two fracture parameters $\left(G_{f}\right.$ and $\left.f_{t}^{\prime}\right)$ is important from the viewpoint of finite element analysis. The R-curve can hardly be used as an input in a general finite element program. Strictly speaking, the R-curve can be a material property only in the limiting sense, for crack extensions from the notch which are negligible compared to both the ligament size and the notch size. For finite crack extensions from the notch, the R-curve depends on the type of loading, geometry of the specimen, and the path of the crack (e.g., straight, inclined, curved). The present theory indeed yields nonidentical R-curves for various situations.

\section{Statistical Analysis of Errors}

To examine the errors, we may construct the plot of $Y=P_{m} / P_{0}$ versus $X=P_{t} / P_{0}$, in which $P_{m}=$ measured $P_{\max }, P_{t}=$ theoretical $P_{\max }$, and $P_{0}$ $=$ failure load based on strength as defined before. With these definitions, it is possible to plot all the data points from Figs. 3-6, whose number is $M=35$ in one figure [Fig. 8(a)]. Furthermore, in Fig. $8(b)$, the same plot is shown for the best results attainable with linear fracture mechanics. Since the ratio $X$ generally decreases as the size of the specimen increases, the points at the top right of the plot refer to small specimens and those at the left bottom of the plot to large specimens.

If the theory were perfect, then the plot of $Y$ versus $X$ would have to be a straight line of slope 1.0, passing through the origin. Thus, the regression line of the plot, $Y^{\prime}=a+b X$, must have a $Y$-intercept close to zero, and slope $b$ close to 1.0 if the fits obtained before are optimum. It is seen from Fig $8(a)$ that this is indeed so. The errors, i.e., the vertical deviations of the data points $Y_{i}(i=1,2,3, \ldots)$ from the regression line may be characterized by the coefficient of variation calculated as

$\omega=\frac{s}{\bar{Y}}, \quad s^{2}=\frac{1}{n-2} \sum_{i=1}^{n}\left(Y_{i}-Y^{\prime}\right)^{2}, \quad \bar{Y}=\frac{1}{n} \sum_{i=1}^{n} Y_{i} \ldots \ldots \ldots \ldots \ldots \ldots$

in which $n=$ number of all data points in the plot; and $s=$ standard error. For the fits from Figs. 3-6, collected in Fig. 8, one obtains

For the present fracture theory [Fig. 8(a)]; $\omega=0.106$;

For linear fracture theory [Fig. $8(b)$ ]: $\quad \omega=0.452$;

For the strength criterion:

$\omega=0.796 \ldots \ldots \ldots \ldots(21)$

The last value for the strength criterion is simply a statistic of the population of $P_{m} / P_{0}$-values. 

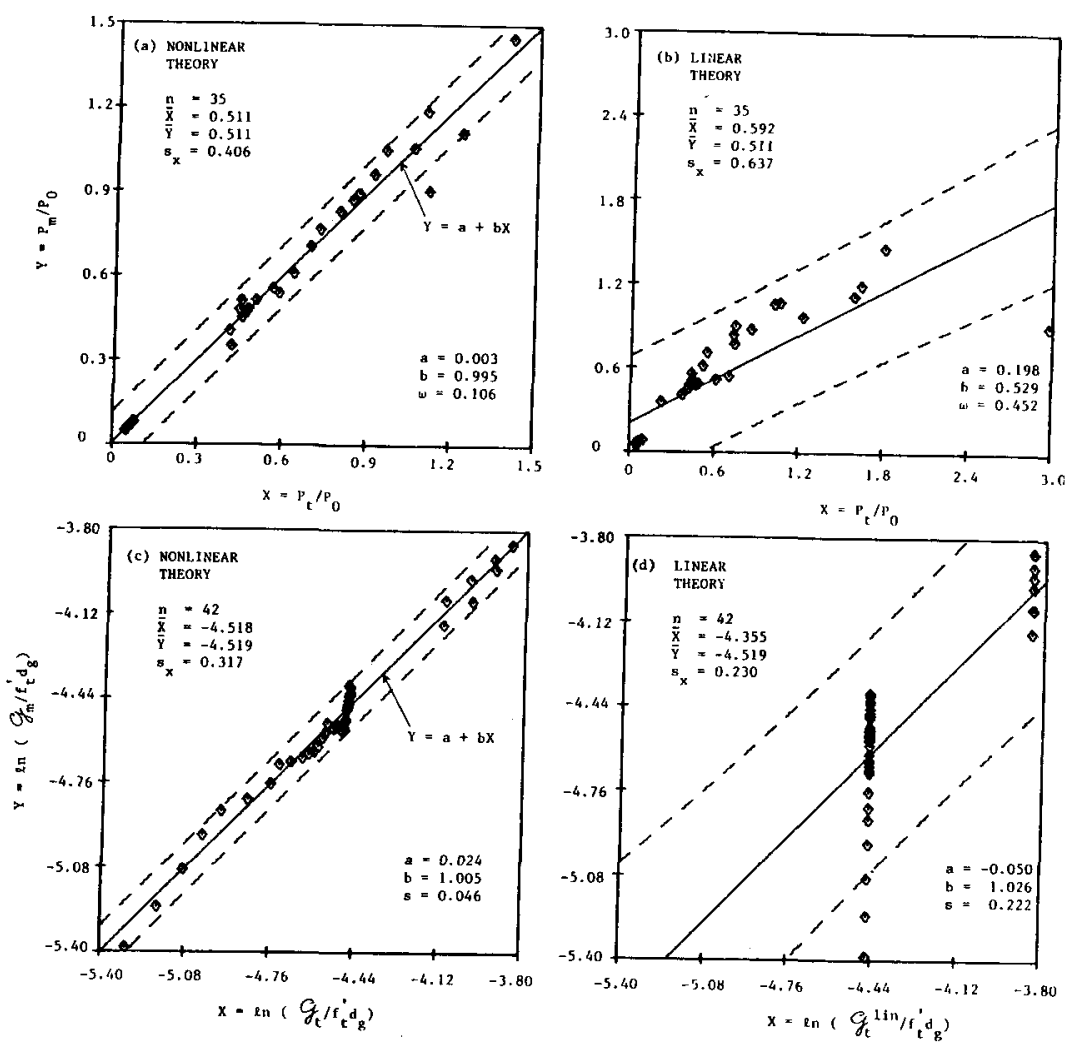

FIG. 8.-Statistical Regression Analysis: $(a, b)$ of Maximum Load Data from Figs. 3-6; and $(c, d)$ of Energy Release Rate Data from Fig. 7

Another possible statistical characteristic is the coefficient of variation of the population of the values of $X=P_{m} / P_{t}$. For all the points in Figs. 3-6 one obtains

For the present fracture theory: $\omega=0.069$;

For linear fracture theory:

$\omega=0.393$.

To carry out a statistical analysis of the errors in the R-curve, the fracture energy values can be normalized with regard to the internal force transmitted by the fracture process zone, which is roughly proportional to $f_{t}^{\prime} d_{g}$ in which $d_{g}=$ the grain size. Accordingly, the comparison may be made in terms of the values of $\ln \left(G_{f} / f_{t}^{\prime} d_{g}\right)$ taken from Fig. 7 . Thus, in Fig. $8(c-d)$, we use on the $Y$-axis the measured values $G_{m}$ of $G_{f}$, and on the $X$-axis we use the theoretical values $G_{t}$ of $G_{f}$. Again, if the theory were perfect, this plot would have to be a straight line $Y^{\prime}=a+b X$ with $a=0$ and $b=1$, and so a linear regression may be applied. The standard error for the vertical deviation from the regression line is then calculated as follows:
For the present fracture theory [Fig. $8(c)$ ]: $s=0.046$;

For linear theory [Fig. 8(d)]:

Fig. 8 also shows the $95 \%$ confidence limits corresponding to $s$ (the dashed lines). These lines are hyperbolas but, due to the relatively large size of our statistical samples, these hyperbolas appear to be almost straight, passing at the vertical distance $\pm 1.96 \mathrm{~s}$ from the regression line.

From this statistical analysis we may conclude that our nonlinear theory achieves a significant improvement over linear elastic fracture mechanics and is capable of satisfactorily describing the available experimental data on rock fracture.

\section{Further Ramifications}

For general finite element analysis, various practical questions arise with regard to the modeling of an inclined or curved crack in the form of a zigzag crack band in the square mesh. In this representation, one must resolve the question of the effective width and length of the zigzag crack band. These questions are analyzed in Refs. 6 and 9.

Another question of importance for general finite element analysis is the modeling of fracture when the direction of the principal stresses and strains in the fracture process zone rotates during the formation of the fracture. This can, for example, happen when a vertical normal stress produces only partial fracturing and the fracture is completed in presence of a superimposed shear stress. For such a case, the secant (total) stress-strain relations used in the present work are not well-suited, and one needs to formulate a tensorially invariant incremental stress-strain relation for tensile strain-softening. One possible formulation is presented in Ref. 4. That work also addresses the question of the fracture energy value when a smoothly curved tensile strain-softening diagram is considered. The problem is approached from the viewpoint of strainlocalization instability, which allows deriving certain simplified expressions for the fracture energy relation to the tensile stress-strain diagram.

It must be emphasized that the present work is limited to Mode I fracture (symmetric, opening mode). In the case of shear fractures or mixed mode fractures, various difficult questions arise with regard to the crack band representation, particularly when the direction of the crack band propagation through the mesh is unknown. These conditions are relegated to subsequent studies.

\section{Conclusions}

1. Due to material heterogeneity, fracture can be modeled by means of a crack band whose front has the width of several times the size of inhomogeneities.

2. The crack band model is convenient for finite element analysis. At its front, the crack band is assumed to have a single-element width, and consequently a proper size of finite elements must be chosen.

3 . The crack band model allows the characterization of fracture by means of stress-strain relations that cover the strain-softening behavior. A simple triaxial form of such stress-strain relations can be formulated 
using the compliance matrix rather than the stiffness matrix.

4. In its simplest form, the crack band model for fracture involves two independent material parameters-the fracture energy and the tensile strength. These two parameters have to be found by fitting fracture test data.

5. The width of the crack band at its front can be taken as five-times the grain size of rock.

6. The present theory is capable of satisfactorily representing all essential fracture test data on rock existing in the literature. In particular, the theory describes well the available maximum load data as well as the measured resistance curves (R-curves).

\section{ACKNOWLEDGMENT}

Partial financial support under Grant No. AFOSR-83-0009 from Air Force Office of Scientific Research to Northwestern University is gratefully acknowledged. Mary Hill is thanked for her invaluable secretarial assistance.

\section{APPENDIX.-RefERENCES}

1. Barenblatt, G. I., "The Formation of Equilibrium Cracks During Brittle Fracture. General Ideas and Hypothesis. Axially-Symmetric Cracks," Prikladnaya Matematika i Mekhanika, Vol. 23, No. 3, 1959, pp. 434-444.

2. Barenblatt, G. I., "The Mathematical Theory of Equilibrium Crack in the Brittle Fracture," Advances in Applied Mechanics, Vol. 7, 1962, pp. 55-125.

3. Bažant, Z. P., "Instability, Ductility and Size Effect in Strain-Softening Concrete," Journal of the Engineering Mechanics Division, ASCE, Vol. 102, No. EM2 Apr., 1976, pp. 331-344.

4. Bažant, Z. P. "Crack Band Model for Fracture of Geomaterials," 4th International Conference on Numerical Methods in Geomechanics, Edmonton, Alberta, June, 1982, (ed. Z. Eisenstein), Proc. Vol. 3 (invited lectures), pp. 1137-1152.

5. Bažant, Z. P., "Microplane Model for Fracture Analysis of Concrete Structures," Proceedings, Symposium on "The Interaction of Non-nuclear Munitions with Structures," U.S. Air Force Academy, Colorado Springs, Colo., May, 1983, pp. 49-55.

6. Bažant, Z. P." "Mechanics of Fracture and Progressive Cracking in Concrete," Report No. 83-2/428m, Center for Concrete and Geomaterials, Northwestern University, Evanston, IIl., Feb., 1983; also Chapt. I in Applications of Fracture Mechanics to Concrete, G. C. Sih, ed., Martinus Nijhoff, The Netherlands, to appear.

7. Bažant, Z. P., and Cedolin, L., "Blunt Crack Band Propagation in Finite Element Analysis," Journal of the Engineering Mechanics Division, ASCE, Vol. 105, No. EM2, Proc. Paper 14529, 1979, pp. 297-315.

8. Bažant, Z. P., and Cedolin, L., "Fracture Mechanics of Reinforced Concrete," Journal of the Engineering Mechanics Division, ASCE, Vol. 106, No. EM6, Proc. Paper 15917, 1980, pp. 1287-1306.

9. Bažant, Z. P., and Cedolin, L., "Finite Element Modeling of Crack Band Propagation in Reinforced Concrete," Report No. 81-9/640f, Center for Concrete and Geomaterials, Northwestern University, Evanston, Ill., 1981.

10. Bažant, Z. P., and Gambarova, P. G., "Rough Cracks in Reinforced Concrete," Journal of the Structural Division, ASCE, Vol. 106, No. ST4, Proc. Paper No. 15330, 1980, pp. 819-842.

11. Bažant, Z. P., and Kim, S. S., "Plastic-Fracturing Theory for Concrete," Journal of the Engineering Mechanics Division, ASCE, Vol. 105, No. EM3, Proc. Paper 14653, 1979, pp. 407-428.
12. Bažant, Z. P., and Oh, B. H., "Crack Band Theory for Fracture of Concrete," Materials and Structures (RILEM, Paris), Vol. 16, 1983, pp. 155-177.

3. Mazant Z P and Oh B. H. "Concrete Fracture via Stress-Strain Relations," Report No. 81-10/665c, Center for Concrete and Geomaterials, Northwestern University, Evanston, III., 1981

14. Bažant, Z. P., and Panula, L., "Statistical Stability Effects in Concrete Failure," Joumal of the Engineering Mechanics Division, ASCE, Vol. 104, 1978, No. EM5, pp. 1195-1212.

15. Bažant, Z. P., and Tsubaki, T., "Slip-Dilatancy Model for Cracked Reinforced Concrete, Journal of the Structural Division, ASCE, Vol. 106, No. ST9, Proc. Paper 15704, 1980, pp. 1947-1966.

16. Carpinteri, A. "Static and Energetic Fracture Parameters for Rocks and Concretes," Report, Istituto di Scienza delle Costruzioni-Ingegneria, University of Bologna, Italy, 1980.

17. Carpinteri, A., "Experimental Determination of Fracture Toughness Parameters $K_{r c}$ and $I_{c}$ for Aggregate Materials," "Advances in Fracture Research," Proc. Paper 5th International Conference on Fracture, Cannes, France, D. Francois, ed., Vol. 4, 1981, pp. 1491-1498.

18. François, ed., Crack Band Analysis," Computer Methods in Applied Mechanics and Engineering, Vol. 24, No. 3, 1980, pp. 305-316.

19. Dugdale, D. S., "Yielding of Steel Sheets Containing Slits," Journal of Mechanics and Physics of Solids, Vol. 8, 1960, pp. 100-104.

20. Evans, R. H. and Marathe, M. S., "Microcracking and Stress-Strain Curves for Concrete in Tension," Matériaux et Constructions, Vol. 1, No. 1, 1968, pp. 61-64.

11. Heilmann H. G. Hilsdorf, H. H., and Finsterwalder, K., "Festigkeit und Verformung von Beton unter Zugspannungen," Deutscher Ausschuss für Stahlbeton, Heft 203, W. Ernst \& Sohn, West Berlin, 1969

22. Hillerborg, A., Modéer, M., and Petersson, P. E., "Analysis of Crack Formation and Crack Growth in Concrete by Means of Fracture Mechanics and Finite Elements" Cement and Concrete Research, Vol. 6, 1976, pp. 773-782.

23. Hoagland, R. G., Hahn, G. T., and Rosenfield, A. R., "Influence of Microstructure on Fracture Propagation in Rock," Rock Mechanics, Vol. 5, 1973, pp. 77-106.

24. Hughes, B. P., and Chapman, G. P., "The Complete Stress-Strain Curve for Concrete in Direct Tension," Bulletin RILEM, No. 30, 1966, pp. 95-97.

25. Ingraffea, A. R., "Numerical Modeling of Fracture Propagation," Report, Cornell University, 1983 (also, to appear in Rock Fracture Mechanics, H. P. Rossmanith, ed. The International Center for Mechanical Sciences, Udine, Italy, 1983)

26. Kfouri, A. P. and Miller, K. J "Stress Displacement Line Integral and Closure Energy Determinations of Crack Tip Stress Intensity Factors," Int. Journal of Pres. Ves. and Piping, Vol. 1, No. 3, 1974, pp. 179-191.

27. Kfouri, A. P., and Rice, J. R., "Elastic/Plastic Separation Energy Rate for Crack Advance in Finite Growth Steps," in "Fracture 1977" (Proc. Paper of the 4th Intern. Conf, on Fracture, held in Waterloo, Ontario), D. M. R. Taplin, ed., University of Waterloo Press, Vol. 1, 1977, pp. 43-59. 28. Knauss, W. G., "On the Steady Propagation of a Crack in a Viscoelastic Sheet;
Experiments and Analysis," Reprinted from the Deformation in Fracture of High Polymers, H. H. Kausch, ed., Plenum Press, 1974, pp. 501-541.

29. Kupfer $\mathrm{H}$ B and Gerstle, K H "Behavior of Concrete under Biaxial Stress," loumal of the Engineering Mechanics Division, ASCE, Vol. 99, No. EM4, Proc. Paper 9917, 1973, pp. 853-866.

30. Liu, T. C. Y. Nilson A. H., and Slate, F. O., "Biaxial Stress-Strain Relations for Concrete," Joumal of the Structural Division, ASCE, Vol. 98, No. ST5, Proc. Paper 8905, 1972, pp. 1025-1034.

31. Laland $\mathrm{K}, \mathrm{Z}$ "Continuous Damage Model for Load-Response Estimation of Concrete," Cement and Concrete Research, Vol. 10, 1980, pp. 395-402. 
32. Lorrain, M., "On the Application of the Damage Theory to Fracture Mechanics of Concrete," A State-of-the-Art Report, Civil Engineering Department, I.N.S.A. 31077 Toulouse, Cedex, France, 1981

33. Mazars, J., "Mechanical Damage and Fracture of Concrete Structures," 5th International Conference on Fracture, D. François, ed., Cannes, France, Vol. 4, 1981, pp. 1499-1506.

34. Ngo, D., and Scordelis, A. C., "Finite Element Analysis of Reinforced Concrete Beams," Journal of the American Concrete Institute, Vol. 64, No. 3, Mar., 1967, pp. 152-163.

35. Parker, A. P., The Mechanics of Fracture and Fatigue, E. F. N. Spon, Ltd., Methuen, London, 1981

36. Petersson, P. E., "Fracture Energy of Concrete: Method of Determination," Cement and Concrete Research, Vol. 10, pp. 78-89, and "Fracture Energy of Concrete: Practical Performance and Experimental Results," Cement and Concrete Research, Vol. 10, 1980, pp. 91-101.

37. Rashid, Y. R., "Analysis of Prestressed Concrete Pressure Vessels," Nuclear Engineering and Design, Vol. 7, No. 4, Apr., 1968, pp. 334-344.

38. Rüsch $\mathrm{H}$ and Hilsdorf, $\mathrm{H}$ "Deformation Characteristics of Concrete Under Axial Tension" Vorunteruchungen Bericht Nr. 44, Munich, May, 1963. der Axial Tension," Voruntersuchungen, Bericht Nr. 44, Munich, May, 1963 39. Schmidt, R. A., "Fracture-Toughness Testing of Limestone," Experimental
Mechanics, Vol. 16, No. 5, 1976, pp. 161-167.

40. Schmidt, R. A., "Fracture Mechanics of Oil Shale-Unconfined Fracture Toughness, Stress Corrosion Cracking, and Tension Test Results," Proceedings, 18th U.S. Symposium Rock Mechs., Paper 2A2, Colorado School of Mines, Golden, Colo., 1977.

41. Schmidt, R. A., and Lutz, T. J., " $K_{I C}$ and $J_{I C}$ of Westerly Granite-Effect of Thickness and In-Plane Dimensions," ASTM STP 678, S. W. Freiman, ed. American Society for Testing and Materials, Philadelphia, Pa., 1979, pp. American Society for Testing and Materials, Philadelphia, Pa., 1979, pp.

42. Suidan, M., and Schnobrich, W. G., "Finite Element Analysis of Reinforced Concrete," Journal of the Structural Division, ASCE, Vol. 99, No. ST10, Oct., 1973 , pp. 2109-2122.

43. Wnuk, M. P., "Quasi-Static Extension of a Tensile Crack Contained in Vis coelastic Plastic Solid," Journal of Applied Mechanics, ASME, Vol. 41, No. 1 1974, pp. 234-248. 